\title{
NUTRITION AND THE INDUSTRIAL WORKER
}

\author{
BY
}

E. H. CAPEL

(From the Medical Department, Joseph Lucas, Ltd., Birmingham)

The subject of nutrition is so vast that it has become impossible for anyone with wide other interests like a medical officer in industry to follow in detail all its ramifications and rapid developments. It is essential, however, that he has an accurate knowledge of the fundamentals.

It is well known that a defective diet will lead to inefficiency and diminished energy as well as deterioration in health and structural changes in the body, just as-for analogy-poor petrol and oil will lead to a bad performance followed by wear and tear and damage to a motor car. This necessity for correct and adequate feeding of industrial workers has been reflected in the Factories (Canteen) Order, 1943, which requires the provision of a canteen in all factories employing more than 250 persons.

The Industrial Medical Officer is interested not only in maintaining health, in the sense of preventing disease, but particularly also in maintaining the maximum working efficiency of the employee. For this reason he must have a knowledge of the bodily requirements, the evidences of malnutrition, especially in minor degrees, and must also have some practical ideas on how to control or improve the dietary of the workers. He must be able to detect malnutrition in new employees and estimate its effects on working ability and the prospects of a cure. He must be able to detect malnutrition arising amongst employees and to determine the factor responsible for it, and he must be in a position to give the management and the canteen manager advice on the quantity, quality and frequency of meals provided for the workers. Also he may have to educate the workers by publicity and personal instruction.

It is proposed, therefore, to go briefly through the main factors necessary to a satisfactory diet and to mention some of the effects of deficiencies. In general these will be confined to the effects of minor deficiency as the effects of major defects are less commonly seen in this country and also are comparatively well known. Lastly, some of the causes of malnutrition will be considered and a few of the problems facing industry will be discussed.

The main constituents of a diet are the following:

1. Water.

2. The energy value (measured in calories).
3. Carbohydrate.

4. Fat.

5. Protein.

6. Salts.

7. Vitamins.

Water is obviously an important part of a diet. It is probable that minor variations do not affect health and it is difficult to state an optimum requirement as this varies with climatic and other conditions. The Factories Act requires a supply of wholesome drinking water at suitable points in the factory. In the case of hot processes when the amount of fluid taken is considerable, attention should be given to the form of fluid, as the habitual ones, tea, beer or barley water, are not always desirable. A suitable saline drink, as mentioned again later, is probably the best.

Energy Value or Calories. The body burns foodstuffs to provide energy, chiefly carbohydrate, fat and protein, but also small amounts of other substances such as alcohol. A certain amount is required for vital processes such as respiration, the circulation and keeping the body warm. This is the basal metabolism and is approximately proportional to the surface area of the body-about 920 calories per square metre. It falls slightly with age. For an adult it usually lies between 1200 and 2000 calories a day, depending on size.

Over and above this, energy is required for work and other activities. The body as a machine compares well with most engines, but even so, is less than 20 per cent. efficient; 5 calories of food eaten produce less than 1 calorie of mechanical energy. Neither is food a cheap fuel. It costs about ten times as much to get mechanical energy from food as from petrol. Or as another example, one unit of electricity costing less than one penny corresponds to 860 calories of which 95 per cent. or more may be converted to mechanical energy. To provide this much mechanical energy from the human body requires more than 4000 calories of food, costing probably twenty times as much. It is thus very expensive to waste human effort and all possible processes should be mechanized.

The calorie requirements of the body vary enormously with different types of work. For example: 
Sitting at rest $\ldots$ requires 30 calories per hour

Standing at rest ..,$\quad 40$, ",

Sweeping the floor .. " 100 ",

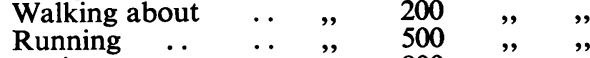

Boxing $\quad . \quad$.. $, \quad, 800 \quad, \quad$,

To generalize one can say light work 50-75, moderate work 75-150, hard work 150-300 and very heavy work 300 or more calories an hour.

From tables such as this calorie requirements of an individual can be fairly accurately worked out. For our purpose this is not necessary and it is usually enough to allow 2400 calories daily for light, sedentary work, 3000-3500 for moderately hard work, rising to 4000 or 4500 for very heavy work.

If a diet is insufficient the weight falls, there is weakness and fatigue and the person feels the cold more than before. After a time the body learns to use food more economically and also bodily movements are economized.

In most cases of starvation other dietary deficiencies will be present as well, showing their characteristic signs and symptoms. When the carbohydrate and fat resources of the body have been used up the body lives on its proteins. This has been suggested as the reason for the lowered resistance to some infections such as tuberculosis.

The provision of an adequate amount of food for energy must always be the first consideration in planning a diet.

Carbohydrate. Carbohydrates supply in a normal diet a very considerable part of the energy requirements of the body. The more complex carbohydrates are broken down to glucose in the intestine before absorption and the glucose then circulates in the blood from which it can either be used directly by the muscles or stored as glycogen in the liver.

Glucose is thus far the most easily assimilable food. There is some evidence that $2-2 \frac{1}{2}$ hours after a meal the blood sugar falls with feelings of fatigue. This tends to be compensated later by an output from the liver. Some athletes claim that glucose taken before a contest improves their performance.

There is often a tendency to condemn carbohydrates. There is good reason to believe that carbohydrates are burned as such by the muscles and that work is done most economically when carbohydrate is the fuel. Provided the other essential dietary factors are adequate the further calorie needs can well be met by carbohydrate.

Fat. Fat is a compact form of energy providing food, one gram being equal to about two of carbohydrate or protein. It is quite probable that fat is not a necessary constituent of a diet as the body can make it out of carbohydrate, though it is possible that it is a necessary vehicle for some of the fat-soluble vitamins. Vitamin A can be produced from the precursor carotene present in vegetables, and vitamin $D$ is formed in the skin by the action of ultra-violet light. Vitamins $\mathrm{E}$ and $\mathrm{K}$ are found in vegetables, and vitamin $K$ is apparently produced in the intestinal tract by the action of certain bacteria, so an outside source may be un- necessary. Two fatty acids-linoleic and linolenic have been found necessary for the health of rats, an absence leading to the development of a skin disease. There is so far no evidence that these fatty acids are necessary for the human, though some vendors of protective skin creams have seized upon it and include linseed oil in their product, together with very exaggerated claims of its merits.

It is probably desirable in the present state of our knowledge to include a certain amount of fat in the diet because of its high calorie value and because of the fat-soluble vitamins. Because the body can obtain the latter by other means it does not necessarily follow that it is always able to do so or to do so in sufficient amounts.

Protein. Proteins are complex organic compounds composed of chains of amino acids. During digestion they are broken down into the separate amino acids and as such absorbed into the body. The body then builds its own protein and probably other substances as well, from these amino acids. The body can apparently synthesize some of the amino acids, but not all. Of the 22 known, 10 are thought to be incapable of synthesis and must be present in the food taken.

Some food contains all or most of these essential amino acids-especially meat, milk and eggs, and comparatively little will supply the needs of the body. These are known as first-class proteins. Others, such as vegetable protein, contain little or none of some of the essential amino acids and hence may only supply bodily needs if taken in large quantities or may not be able to supply them at all. These are known as second-class proteins

Protein is required for the formation of living cells for growth, wear and tear, secretions-milk, mucus, etc., the formation of enzymes, hormones, etc. In addition to the daily intake there is a considerable store of body protein-where is not known for certain, but probably a good deal in the liver.

Protein can be used as fuel, having the same calorie value as carbohydrate, and it is obvious unwanted amino acids must be so used, even on a low protein diet.

Protein requirements are highest during growth and pregnancy, but are also high in alimentary or other diseases in which absorption is defective, and in nephritis, nephrosis, serous effusions, fevers, etc., in which there is excessive loss or destruction of protein.

Total calorie intake has a very important bearing on the protein requirements of the body. With a high calorie diet the protein requirements of an adult can be reduced to quite a low level. If the diet is low in total calories the body has to fall back on the protein to use as fuel, leading to a relative protein deficiency. This is the explanation of most cases of protein deficiency that arise. In almost all cases the total diet is very inadequate.

A normal adult requires $80-100 \mathrm{gm}$. a day, of which at least one-third should be first-class protein. One-and-a-half times this amount is required during pregnancy, lactation and for growth between 14 and 
20 years of age. The effects of deficiency of specific amino acids are not known for certain in the human. The effect of a total deficiency is the production of oedema-famine oedema or nutritional oedema, and like all these things is frequently associated with other nutritional deficiencies.

The symptoms and signs are fatigue, weakness, lessened growth and oedema. Borderline cases may only show oedema after excessive standing, exercise, drinking large quantities of fluid, an excess of salt, getting too hot or other precipitating cause.

It seems probable that some of the cases of oedema we label idiopathic, or postural or Milroys disease may well be nutritional. Confirmation of the diagnosis can be made by a study of the diet and estimation of the blood protein. (Normal serum protein figures total 6-8 gm. per 100 c.c., albumin 4-5.5 gm. per 100 c.c., globulin $1 \cdot 4-3 \mathrm{gm}$. per 100 c.c.) In protein deficiency a reduction of the albumin is particularly significant.

Salts. Although many different salts are important in nutrition the ones that interest us most are sodium, iron and calcium.

Sodium is present in adequate amounts in most diets. Its interest to the industrial medical officer is that workers in hot processes, such as stokers and tinplate workers, lose large quantities of sodium chloride in their sweat, giving rise to weakness, fatigue and cramp in the limbs. Men on these jobs must be provided with plenty of fluid, not as beer or barley water, but as saline, somewhat less than normal strength.

Iron is essential for the formation of haemoglobin. In the breakdown of red cells in the body almost 80 per cent. of the iron is retained, but the other 20 per cent. is lost and must be replaced from food. In growing children and pregnancy new blood is being formed and a greater supply is needed. In women there is a greater loss than in men because of menstruation.

The daily allowance should be $15 \mathrm{mgm}$. for adult men and $20 \mathrm{mgm}$. for adult women, with more during pregnancy. This figure is for available iron. Much of the iron taken, especially that in organic combination, is not absorbed. Inorganic, ferrous iron, is absorbed best. The symptoms and signs of iron deficiency are those of anaemia, and are well known.

Iron deficiency is not, of course, the only cause of anaemia. Lack of vitamin C, as well as the many well-known diseases, can cause failure of haemoglobin formation.

It is worth remembering that the treatment of any appreciable degree of anaemia by diet alone will be very slow. An additional $5 \mathrm{mgm}$. of iron absorbed and used daily only raises the haemoglobin level of the blood by 6 per cent. in a month. Large doses of iron must therefore be given in one of the usual forms such as Pil. ferrous sulph. Diet can only be effective in prevention. Many foods with a high iron content have little available iron-such as meat and milk. Liver, kidneys, nuts, whole grain cereals and treacle are high in available iron.

During pregnancy and when there is a possible dietary iron deficiency medicinal iron should be given as a supplement. All degrees of anaemia should be fully treated to raise the blood to a high normal haemoglobin figure and to obtain good body reserves of iron.

The amount of inefficiency and ill-health caused by even small degrees of anaemia does not seem to be fully appreciated yet it can be so easily treated.

Calcium. There is more calcium in the body than any other mineral element. Most of the calcium is in the bones to which it gives strength and hardness, but a smaller amount is present in the tissues and blood where it serves a number of very essential purposes. It is necessary for the coagulation of the blood, to control neuro-muscular excitability, to control the rhythm of the heart and to control membrane permeability. Normally the body loses about $\frac{1}{2} \mathrm{gm}$. of calcium daily, which must be replaced from the food. If not replaced the calcium will be withdrawn from the bones in an endeavour to keep the blood and tissue calcium up to normal, producing softening of the bones known as osteomalacia. The needs during growth, pregnancy and lactation are much greater.

Calcium cannot be absorbed without an adequate supply of vitamin D and no excess of calcium can make up for its lack. Neither can an excess of vitamin D make up for lack of calcium. It is, for example, useless to treat rickets with vitamin D without also giving adequate calcium. As with most nutritional requirements, some individuals excrete more than others and in some absorption is defective. Absorption of calcium is most likely to be defective in cases of serious gastro-intestinal diseases such as coeliac disease and chronic ulcerative colitis.

As already stated the normal loss of calcium is $0.5 \mathrm{gm}$. daily, so that an adequate diet should contain at least $0.7 \mathrm{gm}$. daily. During pregnancy and lactation 1.5-3 gm. is required and during the years of growth 1-2 gm. daily. Foods vary in the availability of the calcium they contain. Milk is our best source, but even so 1 quart only contains $1 \cdot 25 \mathrm{gm}$.

Calcium in vegetables is poorly absorbed. Diets tending to produce an alkalosis favour the retention of calcium, whereas those tending to produce acidosis tend to favour loss of calcium.

The national loaf is now fortified with calcium carbonate; $7 \mathrm{oz}$. of calcium carbonate is added to each 280-lb. sack of flour. It has been shown by Harrison and Mellanby, and McCance and Widdowson that the considerable amount of phytic acid present in national flour interferes with the absorption of calcium and for this reason the adding of calcium to the flour is desirable. Some writers disagree with this view and argue that there is no evidence of calcium deficiency in the population and that we risk the danger of an excess of calcium which may cause urinary calculi, increased blood pressure and interfere with kidney and heart functions. In any case the amount added is very small and seems unlikely to do any appreciable harm. 
The relation between lead and calcium metabolism is of special industrial interest. Lead absorbed by the body is stored in the bones. Conditions favouring the deposition of calcium in the bones favour the fixing of lead in the bones, and conditions leading to decalcification such as excessive calcium loss or deficient intake or absorption mobilize lead from the bones and may precipitate an attack of lead poisoning. In the case of lead workers, therefore, it is wise policy to give a high calcium diet either as additional milk or by giving calcium lactate, and so fix some of the absorbed lead to prevent the production of symptoms. There may, of course, be occasions when it is desirable to mobilize this lead from the bones and remove itto 'de-lead' the patient. This must be done under controlled conditions and when the patient is not working.

\section{The Vitamins}

The vitamins are a group of complex chemical substances required by the body for carrying on certain metabolic processes. Some can be made by the body itself, but others must be provided in the diet. The subject is confusing because of the multiple, undescriptive and frequently changing nomenclature. Just prior to the war over 3000 original papers on the vitamins appeared annually, so that it disconcerts even the specialists. It is proposed, therefore, to speak only of the better understood vitamins and their main practical aspects.

Vitamin A. Vitamin A is a fat soluble substance present only in animal fats. It can be formed in the body from carotene, a colouring substance present in most vegetables. Vitamin A is essential to growth and health-in particular the health and nutrition of epithelial cells and to the formation of visual purple in the retina. Its alleged anti-infective properties are probably only related to the maintenance of healthy epithelium.

Deficiency leads to atrophy of epithelial cells and conversion of more specialized epithelium to stratified epithelium. The failure to form visual purple produces night blindness. Severe degrees of deficiency lead to the disease Xerophthalmia. This disease begins with photophobia, itching and burning of the eyes and slight conjunctivitis. The eyes become dry, the conjunctiva pigmented and the cornea oedematous and infiltrated with leucocytes. This is usually followed by secondary infection with ulceration and destruction of the eye.

The lesser degrees of deficiency which interest us most lead to changes in the skin which becomes dry, rough and slaty in colour with a papular eruption due to hyperkeratosis of the hair follicles, giving a toad skin appearance. The changes start on the anterolateral surface of the arms and legs and spread over the abdomen, buttocks, back and neck. Some degree of night blindness also occurs and is probably the easiest sign to detect, and a light adaptation test is the most reliable means of determining vitamin A deficiency.
Diagnosis of vitamin A deficiency is made from a study of the diet, the clinical findings and the light adaptation test.

The normal daily requirement is about 4000 international units. The most reliable sources in food, apart from fish liver oils, are liver, egg yolk, cheese and butter. The precursor carotene is best supplied in the outer leaves of green vegetables and in carrots. There is sometimes difficulty in absorbing carotene, especially in certain diseases, or difficulty in converting it to vitamin $\mathbf{A}$ if absorbed. Diabetes is a case in point where carotene cannot be converted to vitamin $\mathrm{A}$ and in the high vegetable diets sometimes taken becomes deposited in the skin giving the well-known orange pigmentation.

Vitamin A is better given, therefore, as vitamin A and not as carotene, though as a result of the war and shortage of animal fats it may be necessary for us to rely more and more on that provided from vegetables.

Vitamin D. Vitamin D consists of a group of fat-soluble substances-activated sterols-closely related to cholesterol. It occurs in both animal and vegetable fats and in addition can be formed in the body by the action of sunlight on the skin. It is necessary for the proper absorption of calcium and phosphorus and in the formation of bone in the body. The normal body requirement is $800-1200$ units daily, though some writers believe considerably less than this to be sufficient.

During growth, pregnancy and lactation, when the calcium metabolism is so much higher than at other times, the need for vitamin D is considerably greater and it is consequently at these times that deficiency most commonly becomes manifest. A deficiency during growth leads to rickets. In adults it leads to decalcification of the bones and the disease osteomalacia. Symptoms of tetany may occur in either of these diseases due to the lowered blood calcium. Osteomalacia seldom occurs except during pregnancy and then is rare except in famines.

The best sources of supply are fish liver oils, egg yolk, salmon, sardines, butter and cream. The ordinary adult can get an adequate supply by a reasonable amount of sunlight and it seems that at present we need not worry much about a possible deficiency except during growth, pregnancy and lactation.

Vitamin C. Ascorbic acid is a water soluble substance present in most fruit and vegetables and essential to the body for health. It cannot be formed in the body and is poorly stored so that a regular daily supply is essential. It is necessary for the formation of intercellular ground substance and especially for the maintenance of intact blood vessels and the formation of collagen.

Advanced deficiency of ascorbic acid produces the well-known disease of scurvy with weakness, pains in the limbs, haemorrhage and stomatitis.

The less serious degrees of deficiency which interest us more are less definite in their picture, but can lead to a considerable amount of reduced effciency and minor ill-health. The symptoms pro- 
duced include fatigue, anaemia, slight oedema, sore mouth, delayed wound healing and possibly a lowered resistance to infection.

Vitamin C deficiency can be fairly accurately estimated by the saturation test in which a large dose of ascorbic acid is given followed by estimation of the amount excreted in the urine. A person deficient in vitamin $\mathrm{C}$ will excrete considerably less than a person not deficient. Investigations carried out on these lines have suggested that there might be a good deal of minor deficiency in the community, especially during spring and early summer.

The normal requirements for an adult lie between $50 \mathrm{mgm}$. and $100 \mathrm{mgm}$. of ascorbic acid daily.

Our main source of ascorbic acid is fruit, potatoes and other vegetables, and during spring and early summer when fruit is not available potatoes and greens supply most. One important point to remember is the ease with which it is destroyed or lost. Prolonged cooking destroys vitamin C. Prolonged soaking washes it out and it is usually thrown down the drain. Vegetables should therefore be covered with the minimum of water and this water not wasted. Also ascorbic acid is much more stable in acids than in alkalis and the use of cooking soda is therefore detrimental.

Bearing these points in mind and with the supplies at present available there should be no difficulty in providing a diet adequate in vitamin $\mathrm{C}$.

\section{The Vitamin B Complex}

What was once known as vitamin $B$ has since 1926 been split up into well over a dozen different substances having different names and actions. I propose only to mention the three better understood fractions-vitamin $B_{1}$, ribo-flavine and nicotinic acid.

Vitamin $\mathbf{B}_{1}$. Vitamin $\mathbf{B}_{\mathbf{1}}$, Aneurin or Thiamin is a water-soluble substance easily destroyed by heat. It is poorly stored by the body and the body is unable to make it itself. It is essential to the body for carbohydrate metabolism so that the amount required depends on the amount of carbohydrate in the diet. It is also necessary for maintenance of normal nerves, appetite and digestion, circulation and growth.

Serious deficiency leads to the disease beriberi with peripheral neuritis, oedema and heart failure. The manifestation of less severe degrees of deficiency are fatigue on slight exertion, loss of appetite, tenderness of the calves and later progressive weakness, nausea, vomiting and tachycardia. These symptoms, like those of other vitamin deficiencies, are not very characteristic, but they have been determined by careful experiment and respond very rapidly to treatment with vitamin $\mathbf{B}_{1}$.

The best dietary sources are meat, liver, the germ of cereals, peas, beans and yeast. The normal daily requirements may be taken as 1-3 mgm. or 300-900 international units, though the requirements, of course, are related to the carbohydrate in the diet. It has been found that beriberi only occurs if the ratio $B_{1}$ (units)/non-fat calories in diet is less than $0 \cdot 12$.
The diagnosis of $B_{1}$ deficiency will depend on the clinical picture, diet history and a therapeutic test.

Riboflavine (Vitamin $\mathbf{B}_{2}$ or $\mathbf{G}$ ). Riboflavine is a water-soluble, heat-resistant substance first isolated in 1932, although the clinical picture of the deficiency syndrome was not described until 1938. It appears to be essential for carbohydrate metabolism, for normal growth and for the health of epithelial tissue.

Riboflavine deficiency gives rise to the following clinical manifestations. First cheilosis-or angular stomatitis-in which the angles of the mouth develop bilateral transverse fissures, a condition we previously knew as perleche. The lips themselves become abnormally red and shiny due to superficial denudation of the epithelium and later cracked and crusted with blood. In addition to cheilosis the skin shows changes resembling seborrhoeic dermatitis with a scaly, greasy desquamation of the naso labial folds, which later may involve the alae nasi, bridge of nose, eyebrows, ears and other parts of the body. Besides these changes the eyes show vascularization of the cornea and conjunctival congestion. The first sign is circum corneal injection which, if not visible to the naked eye, is visible with a slit lamp and shows vessels beginning to invade the cornea.

Riboflavine is widely distributed in foodstuffs, but amongst the best sources are yeast, milk, eggs, fish roe, kidney, liver, heart and growing leafy vegetables.

The normal daily requirements are not yet accurately known but $1-2 \mathrm{mgm}$. is suggested.

Nicotinic Acid. Nicotinic acid is a water soluble heat-stable vitamin, related chemically, but not pharmacologically to nicotine. It is essential to the body for growth and for health of epithelial and nervous tissue. Like vitamin $\mathbf{B}_{1}$ and riboflavine it appears to be necessary for carbohydrate metabolism.

Gross deficiency causes pellagra, a disease characterized by a sore tongue, dermatitis and mental changes leading to insanity.

Minor degrees of deficiency are probably not uncommon. The symptoms are ill-defined and include abdominal pains, indigestion, lassitude and nervous and mental depression. A sore mouth and tongue is one of the commonest symptoms. The tongue shows first a marginal atrophy and later becomes red raw and glazed. Infection with Vincents angina is common and it has been suggested that Vincents angina can only attack tissues weakened by nicotinic acid deficiency.

The nervous symptoms are important to remember as it seems quite possible that this may be the explanation of many of the vague cases of depression. debility, and doubtful psychoneurosis we see.

At present the diagnosis must rest mainly on symptomatology, diet history and on therapeutic tests. Therapeutic tests are very easily applied and the response very rapid.

Nicotinic acid, like other components of the B complex, is widely distributed in food, particularly 
in meat products such as liver, eggs, salmon and in whole cereals and yeast.

The probable daily requirement is about 20 $50 \mathrm{mgm}$.

Of the other vitamins which have been and are being discovered it is not proposed to say anything except that if we keep to a good mixed diet, adequate to provide the ones mentioned, it is improbable that the others will be grossly deficient.

Should evidence of malnutrition appear in a community the factors responsible will probably be found under one of the following main headings:

1. Insufficient food available-insufficient variety or faulty distribution. Poverty-insufficient money to buy the food.

2. Faulty likes and habits of the people. This is closely linked with what shops sell, canteen and restaurants provide and publicity in and out of the factory. Also legislation-e.g. national bread.

3. Faulty cooking, preparation and treatment of the food.

4. Diseases and abnormalities of absorption in the individual.

Are there nutritional deficiencies occurring in this country now and, if not, are there prospects of them arising? It would take too long to discuss all the arguments for and against these points now, but it seems safe to say that, except in special cases such as badly fed institutions, and in people with deficiencies of absorption or other predisposing disease, the gross deficiency diseases do not occur. There does seem, however, to be evidence of much minor malnutrition-not necessarily worse than in prewar days-but sufficient and sufficiently widespread to be a cause of much minor ill-health and loss of efficiency. There may be many and real difficulties in giving a fully optimum diet to all in war time, but there is no doubt that by careful distribution, the provision of sensible meals, and most of all the education of workpeople and others in correct habits of diet, likes and dislikes, cooking, etc., much improvement can be made. It must, of course, be emphasized that malnutrition is not the cause of all minor ill-health-probably only of a small part. All the other factors-fatigue, ventilation, exercise, psychological considerations-must still be given their proper attention.

In addition to these considerations certain practical aspects of the problem of feeding workpeople arise.

The first is what meals and at what frequency should meals be provided at the factory? It would seem probable that a meal or snack every $2 \frac{1}{2}$ or 3 hours is the most desirable. This should help to maintain efficiency by preventing a fall in blood sugar which may occur several hours after a meal, and it might help also to reduce the incidence of peptic ulcer which is at present very high and a cause of much disability and absenteeism. It is suggested, therefore, that facilities should be available for obtaining (1) breakfast for those who have a long journey to work ; (2) a mid-morning snack; (3) a midday full meal; (4) an afternoon snack or tea; (5) an evening meal for those with long travelling or not wishing to go straight home. Equivalent arrangements for feeding night workers should be arranged. Many night workers, especially those on alternating day and night shifts, take normal daytime meals and very little food during the working hours. They should be encouraged to eat during the working hours and sleep in the day, and good canteen facilities will be an incentive to do so.

The food value of the meals provided is of some importance. Inasmuch as the midday meal is the main meal of the day for most people it should provide $1000-1500$ calories, two-thirds or more of the vitamin and mineral salt requirements and a considerable proportion-at least one-third-of the protein requirements. This is the standard aimed at by British restaurants. Whilst it might be desirable to have some of these factors more evenly distributed over the other meals, one must remember that it is the habit of many to have bread and butter, bread and lard or bread and margarine as almost their only other food. Other canteen meals may be mainly calorie producing, though opportunity should be taken to introduce vitamins, salts and proteins when possible.

The canteen should also aim at providing special diets for certain persons suffering from diseases such as peptic ulcer, diabetes and cholecystitis. Special consideration should be given to the meals available for juveniles in view of their high requirements of protein, calcium, iron and vitamins, and if possible they should be provided free or cheaply.

In addition to providing sound meals it will be found necessary to encourage people to use the canteen. To do this the meals must be cheap, well cooked, soundly chosen and attractive, and the canteen conditions must be pleasant and comfortable. Publicity on the principles of sound nutrition should also be undertaken by the normal channels of posters, pamphlets and lectures, and individual instruction when occasion arises should be given by the nurse or medical officer.

The medical officer should co-operate with the canteen manager in arranging the type and frequency of meals provided and may help in the construction of menus, both for normal and special diets. He should also keep him acquainted with the latest developments in nutrition, including certain scientific aspects of cooking.

In conclusion, let it be said that many difficulties stand in the way of fully applying the scientific principles of nutrition, chief of which are oldstanding habits and tradition. Progress will at the best be slow and patient education of the workers themselves, the factory management and the canteen staff will be necessary if results are to be obtained. 